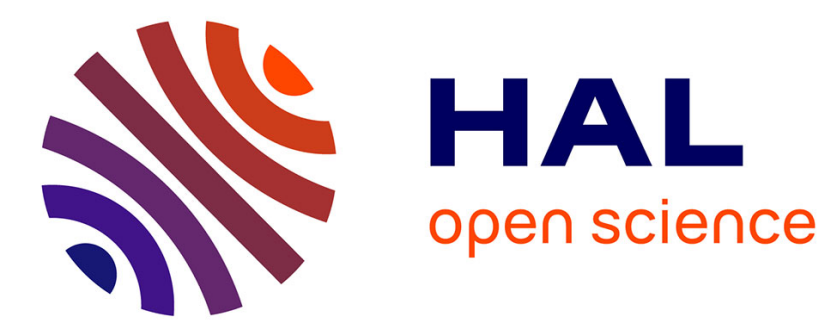

\title{
The logical encoding of Sugeno integrals
}

\author{
Didier Dubois, Henri Prade, Agnès Rico
}

\section{To cite this version:}

Didier Dubois, Henri Prade, Agnès Rico. The logical encoding of Sugeno integrals. Fuzzy Sets and Systems, 2014, vol. 241, pp. 61-75. 10.1016/j.fss.2013.12.014 . hal-01123494

\section{HAL Id: hal-01123494 https://hal.science/hal-01123494}

Submitted on 5 Mar 2015

HAL is a multi-disciplinary open access archive for the deposit and dissemination of scientific research documents, whether they are published or not. The documents may come from teaching and research institutions in France or abroad, or from public or private research centers.
L'archive ouverte pluridisciplinaire HAL, est destinée au dépôt et à la diffusion de documents scientifiques de niveau recherche, publiés ou non, émanant des établissements d'enseignement et de recherche français ou étrangers, des laboratoires publics ou privés. 


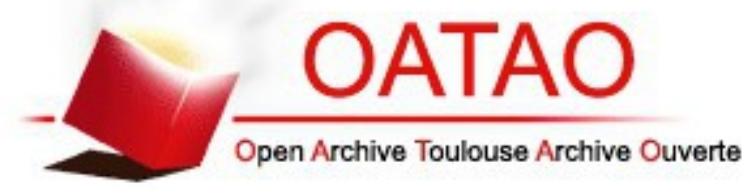

\section{Open Archive TOULOUSE Archive Ouverte (OATAO)}

OATAO is an open access repository that collects the work of Toulouse researchers and makes it freely available over the web where possible.

This is an author-deposited version published in : http://oatao.univ-toulouse.fr/ Eprints ID : 12975

To link to this article : DOI :10.1016/j.fss.2013.12.014

URL : http://dx.doi.org/10.1016/j.fss.2013.12.014

To cite this version : Dubois, Didier and Prade, Henri and Rico, Agnès The logical encoding of Sugeno integrals. (2014) Fuzzy Sets and Systems, vol. 241. pp. 61-75. ISSN 0165-0114

Any correspondance concerning this service should be sent to the repository administrator: staff-oatao@,listes-diff.inp-toulouse.fr 


\title{
The logical encoding of Sugeno integrals
}

\author{
Didier Dubois ${ }^{\mathrm{a}}$, Henri Prade ${ }^{\mathrm{a}}$, Agnès Rico ${ }^{\mathrm{b}, *}$ \\ a Institut de Recherche en Informatique de Toulouse IRIT, Université de Toulouse, 118 route de Narbonne, 31062 Toulouse Cedex 09, France \\ b Equipe de Recherche en Ingénierie des Connaissances ERIC, Université Claude Bernard Lyon 1, \\ 43 bld du 11 novembre 69100 Villeurbanne, France
}

\begin{abstract}
Sugeno integrals are a well-known family of qualitative multiple criteria aggregation operators. The paper investigates how the behavior of these operators can be described in a prioritized propositional logic language, namely possibilistic logic. The case of binary-valued criteria, which amounts to providing a logical description of the fuzzy measure underlying the integral, is first considered. The general case of a Sugeno integral when critersia are valued on a discrete scale is then studied.
\end{abstract}

Keywords: Discrete Sugeno integrals; Possibilistic logic; Possibility theory

\section{Introduction}

Simple multiple criteria aggregation attitudes are rather easy to grasp. Thus, a strict conjunctive attitude based on the minimum operator amounts to saying that the global evaluation reflects the worst rating among local evaluations according to each criterion. With an averaging attitude between two criteria, a global medium score corresponds to having either both criteria half satisfied or one poorly satisfied and the other one fully satisfied. When more sophisticated aggregation operators are used, it becomes difficult to figure out what is their exact meaning from a preference representation point of view, and more generally to provide explanations on the reasons why an object receives some global score [18].

In this paper, we consider an important family of qualitative aggregation operators, namely Sugeno integrals $[23,24]$. They range between minimum and maximum operators, and behave more generally as the median of the normalized ratings according to the various criteria, together with importance levels of groups of best satisfied criteria. In Sugeno integrals, each subset of criteria is indeed associated with an importance level, which enables some synergy between them to be modeled. Sugeno integrals have found many applications in multi-criteria decision aid [15]. In the following we investigate the possibility of providing an equivalent logical representation of Sugeno integrals in the setting of possibilistic logic where classical logic propositions are associated with priorities.

The contribution of the paper is first to provide a set of results for converting Sugeno integrals into two kinds of possibilistic logic formats. Another contribution is to show that our encoding comes close to a previous proposal by Greco et al. [16] using the so-called single-graded rules. These results may shed some light on the intended meaning of the aggregation, in logical terms, for instance when Sugeno integrals are obtained by means of a learning

\footnotetext{
* Corresponding author.

E-mail addresses: dubois@irit.fr (D. Dubois), prade@irit.fr (H. Prade), agnes.rico@univ-lyon1.fr (A. Rico).
} 
procedure $[26,25]$ from observed data describing how people associate a global evaluation to elementary ones. Indeed, we may then provide some explanations on the evaluation procedure used by the persons supplying the data, showing them which rules they implicitly followed.

The paper ${ }^{1}$ is structured as follows. The next section provides notations and background regarding discrete Sugeno integrals on the one hand, and possibilistic logic on the other hand. Section 3 then discusses the logical representation of a fuzzy measure, which corresponds to a global evaluation based on binary valued criteria. Section 4 deals with the general case of a Sugeno integral when criteria are graded on a discrete bounded scale. Section 5 discusses related works and especially the one by Greco et al. [16], before concluding.

\section{Notations and background}

We consider a set $\mathcal{P}=\{1, \ldots, n\}$ of criteria (properties) and a set $X$ of alternatives evaluated according to each criterion. We suppose that all criteria share the same evaluation scale, a finite totally ordered set $L$ with $k+1$ levels denoted by $0=\lambda_{1}<\cdots<\lambda_{k+1}=1$. Each alternative $x \in X$ is encoded as a vector of ratings $x=\left(x_{1}, \ldots, x_{n}\right) \in L^{n}$ where $x_{i}$ is the rating of $x$ with respect to criterion $i$.

The aim of this paper is to bridge the gap between two objects of different nature, introduced in different information modeling perspectives, namely discrete Sugeno integrals and possibilistic knowledge bases. We now recall the necessary background on both of them successively.

\subsection{Discrete Sugeno integral}

The discrete Sugeno integral is used in qualitative decision theory and multiple criteria decision-making in order to synthesize the partial ratings of an object into a global evaluation. Its definition is based on fuzzy measures [23], also called capacities after Choquet [3].

Definition 1. A fuzzy measure, or capacity, $\mu: 2^{\mathcal{P}} \rightarrow L$ is an increasing set function (in the wide sense: $\mu(T) \leq \mu(S)$ if $T \subseteq S \subseteq \mathcal{P}$ ) such that $\mu(\emptyset)=0$ and $\mu(\mathcal{P})=1$.

The fuzzy measure $\mu$ defines an importance system on groups of criteria. In the following, for criteria $i, j, k$, importance levels $\mu(\{i\})$ and $\mu(\{j, k\})$ are respectively denoted by $\mu_{i}$ and $\mu_{j, k}$ for short. Clearly, $\mu_{i, i}=\mu_{i}, \mu_{j, k}=\mu_{k, j}$ and more generally, $\mu_{i, j, \ldots, r}=\mu_{\sigma(i), \sigma(j), \ldots, \sigma(r)}$ for any permutation $\sigma$ of the indices. The increasingness of the fuzzy measure ensures that $\forall i, j, \ldots, r, \mu_{i} \leq \mu_{i, j} \leq \mu_{i, j, \ldots, r}$.

Capacities can represent synergies among criteria. For instance, the case when $\max \left(\mu_{i}, \mu_{j}\right)<\mu_{i, j}$ expresses that criteria $i$ and $j$ are not redundant, and together they have a higher importance than any individual one, which can be viewed as a form of positive synergy between them. More generally, if $\mu(T)>\max _{S \subset T} \mu(S)$, it means that the group $T$ of criteria brings about some strength none of its subsets possesses. A capacity such that $\mu(T)=\max _{S \subset T} \mu(S)$ expresses the case where some criteria in $T$ are redundant, even if of various individual degrees of importance. When $\mu(T)=\max _{i \in T} \mu_{i}$ (this is the maxitivity property), criteria are individually redundant and $\mu$ represents a possibility measure [8], as discussed in the next subsection. In that case, knowing the importance degrees of single criteria is enough to reconstruct $\mu$, and $\mu_{i}=1$ for at least one $i$. The mapping assigning degree $\mu_{i}$ to each $i=1, \ldots, n$ is then called a possibility distribution, often denoted by $\pi$.

Remark. In numerical approaches to criteria aggregation using Choquet integrals (see, e.g., [15]), weights of elementary criteria can be added, and the positive synergy between two criteria $i, j$ is modeled by a super-additive weight $\mu_{i, j}>$ $\mu_{i}+\mu_{j}$. Independent criteria are such that $\mu_{i, j}=\mu_{i}+\mu_{j}$. A qualitative approach cannot express this kind of independence. However, in the qualitative approach $\mu(T)$ represents a kind of importance level of the group $T$ of criteria, but it acts as a threshold, not as a weight. In this context, where importance levels can only be compared, the inequality $\max \left(\mu_{i}, \mu_{j}\right)<\mu_{i, j}$ can thus be interpreted as a form of positive synergy, understood as non-redundancy, while in the numerical setting with weights, the case when $\max \left(\mu_{i}, \mu_{j}\right)<\mu_{i, j}<\mu_{i}+\mu_{j}$ corresponds to a negative synergy.

\footnotetext{
${ }^{1}$ It is a fully revised and extensively rewritten version of a workshop paper [12] with new results.
} 
Given a fuzzy measure, we can compute its qualitative Möbius transform which contains the minimal amount of information sufficient to reconstruct the fuzzy measure.

Definition 2. The qualitative Möbius transform [21,14] of the fuzzy measure $\mu$ is a set-function $\mu_{\#}$ on $\mathcal{P}$ defined by

$$
\forall T \subseteq \mathcal{P}, \quad \mu_{\#}(T)= \begin{cases}\mu(T), & \text { if } \mu(T)>\max _{S \subset T} \mu(S) \\ 0, & \text { otherwise }\end{cases}
$$

It can be checked that

$$
\forall T \subseteq \mathcal{P}, \quad \mu(T)=\max _{S \subseteq T} \mu_{\#}(S) .
$$

In analogy with the theory of belief functions [22], the sets $T$ such that $\mu_{\#}(T) \neq 0$ are called the focal sets of $\mu$ and form the set $\mathcal{F}_{\mu}$. Note that contrary to a usual mass function in belief function theory, the values $\mu_{\#}(T)$ are strictly increasing under inclusion on $\mathcal{F}_{\mu}$. Moreover, $\mu_{\#}(T)=1$ for at least one focal set $T$. $\mu_{\#}$ can thus be viewed as a possibility distribution on the set of subsets of criteria. It coincides with a possibility distribution over singletons if and only if the fuzzy measure is maxitive. The set of focal sets represents all groups of criteria that are not redundant as a whole.

The Sugeno integral of an alternative $x$ with respect to the fuzzy measure $\mu$ is often defined as follows [23]:

\section{Definition 3.}

$$
\oint_{\mu}(x)=\max _{i=1}^{k+1} \min \left(\lambda_{i}, \mu\left(X_{\lambda_{i}}\right)\right)
$$

where $\forall \lambda \in L, X_{\lambda}=\left\{i \in \mathcal{P} \mid x_{i} \geq \lambda\right\}$.

When $x$ is a Boolean alternative $\left(x_{i} \in\{0,1\}\right), \oint_{\mu}(x)=\mu\left(T^{x}\right)$ where $T^{x}=\left\{i, x_{i}=1\right\}$ is the set of criteria satisfied by $x$. This equality provides a simple interpretation of the values of the fuzzy measure as the global evaluation of objects fully satisfying some criteria and fully violating other ones. The above expression makes it clear that $\oint_{\mu}(x)$ is the median value in the set $L \cup\left\{\mu\left(X_{\lambda_{i}}\right), i=2, \ldots, k+1\right\}$ [17].

The Sugeno integral can be equivalently written under various forms $[23,24,9,19,20]$ :

\section{Proposition 1.}

$$
\begin{aligned}
\oint_{\mu}(x) & =\max _{T \subseteq \mathcal{P}} \min \left(\mu(T), \min _{i \in T} x_{i}\right)=\min _{T \subseteq \mathcal{P}} \max \left(\mu(\mathcal{P} \backslash T), \max _{i \in T} x_{i}\right) \\
& \left.=\max _{i=1}^{k+1} \min \left(\lambda_{i}, \mu\left(X_{\lambda_{i}}\right)\right)=\min _{i=1}^{k+1} \max \left(\lambda_{i}, \mu\left(X_{\lambda_{i+1}}\right)\right) \text { (where } X_{\lambda_{k+2}}=\emptyset\right) .
\end{aligned}
$$

Note that the knowledge of the qualitative Möbius transform is enough to calculate the Sugeno integral in the form

$$
\oint_{\mu}(x)=\max _{T \subseteq \mathcal{F}_{\mu}} \min \left(\mu_{\#}(T), \min _{i \in T} x_{i}\right)
$$

Remark. We cannot write $\oint_{\mu}(x)=\oint_{\mu_{\sharp}}(x)$ without ambiguity as the equality $\max _{i=1}^{k+1} \min \left(\lambda_{i}, \mu\left(X_{\lambda_{i}}\right)\right)=$ $\max _{i=1}^{k+1} \min \left(\lambda_{i}, \mu_{\#}\left(X_{\lambda_{i}}\right)\right)$ generally does not hold.

When the evaluation scale is the unit interval $[0,1]$, it has been noticed $[19,6]$ that Sugeno integral coincides with idempotent lattice polynomials, that is, functions $P\left(x_{1}, \ldots, x_{n}\right)$ defined by combining variables $x_{1}, \ldots, x_{n}$ (representing the degrees of fulfillment of $n$ properties), and constants $c_{1}, \ldots, c_{m}$ in [0,1], with min, max, and parentheses, and such that the limit conditions $P(1, \ldots, 1)=1$ and $P(0, \ldots, 0)=0$ hold (see also [4] in the more general case of distributive lattices). Thus, Boolean combinations of properties, involving no negation, are particular cases of Sugeno integrals. 
This suggests that Sugeno integrals may be viewed not only as an evaluation tool, but as a knowledge representation tool as well.

We now present a reminder on two dual representation possibilistic logic formats that we want to relate to Sugeno integrals.

\subsection{Possibilistic logic: conjunctive and disjunctive normal forms}

Consider a propositional language $\mathcal{L}$ containing formulas denoted by $\varphi$, $\psi$, etc. The logical negations, conjunctions and disjunctions are respectively denoted by $\neg, \wedge$ and $\vee$. Let $\Omega$ be the set of interpretations of $\mathcal{L}$. Let $M(\varphi)$ be the set of models of $\varphi$, the interpretations where $\varphi$ is true.

\subsection{1. $N$-bases}

A possibilistic logic base $B^{N}$ is a set of pairs $\left\{\left(\varphi_{j}, \alpha_{j}\right) \mid j=1, \ldots, m\right\}$ where $\varphi_{j} \in \mathcal{L}$ is a propositional logic formula and $\alpha_{j} \in L$ is a priority level [10]. We assume that $L$ is equipped with its order-reversing map that we denote by $1-\cdot: 1-\lambda_{i}=\lambda_{n-i+1}$. Each formula $\left(\varphi_{j}, \alpha_{j}\right)$ means that $N\left(\varphi_{j}\right) \geq \alpha_{j}$ where $N$ is a necessity measure, ${ }^{2}$ i.e., a capacity satisfying the property $N(\varphi \wedge \psi)=\min (N(\varphi), N(\psi))$. A necessity measure is associated to a possibility distribution $\pi$, a mapping $\Omega \rightarrow L$, as follows:

$$
N(\varphi)=\min _{\omega \notin M(\varphi)}(1-\pi(\omega)) .
$$

The capacity $\Pi(\varphi)=1-N(\neg \varphi)=\max _{\omega \in M(\varphi)} \pi(\omega)$ is the possibility measure associated to $N$.

At the semantic level, to the base $B^{N}$ is associated the least informative possibility distribution on the set of interpretations induced by the constraints $N\left(\varphi_{j}\right) \geq \alpha_{j}$ for $j=1, \ldots, m$ :

$$
\pi_{B}^{N}(\omega)=\min _{j=1}^{m} \pi_{\left(\varphi_{j}, \alpha_{j}\right)}(\omega) \text { where } \pi_{\left(\varphi_{j}, \alpha_{j}\right)}(\omega)= \begin{cases}1, & \text { if } \omega \in M\left(\varphi_{j}\right) \\ 1-\alpha_{j}, & \text { if } \omega \notin M\left(\varphi_{j}\right) .\end{cases}
$$

An interpretation $\omega$ is all the more possible as it does not violate any formula $\varphi_{j}$ having a higher priority level $\alpha_{j}$. Hence, this possibility distribution is expressed as a min-max combination of the form:

$$
\pi_{B}^{N}(\omega)=\min _{j=1}^{m} \max \left(1-\alpha_{j}, I_{M\left(\varphi_{j}\right)}(\omega)\right)
$$

where $I_{M\left(\varphi_{j}\right)}$ is the characteristic function of $M\left(\varphi_{j}\right)$.

If the necessity measure $N$ is based on the possibility distribution $\pi$, it holds that $N\left(\varphi_{j}\right) \geq \alpha_{j}, \forall j=1, \ldots, m$ if and only if $\pi \leq \pi_{B}^{N}$. So, the possibilistic base $B^{N}$ is a description of a possibility distribution "from above".

A possibilistic base $B^{N}$ can be always rewritten as a base where the formulas $\varphi_{i}$ are clauses (without altering the distribution $\pi_{B}^{N}$ ), thanks to the decomposability of necessity measures with respect to conjunction. Indeed, any formula $\varphi \in L$ can be written as a conjunction $\varphi \equiv \wedge_{j=1}^{\ell} \psi_{j}$ where $\psi_{j}$ is a clause. And $(\varphi, \alpha)$ corresponds to the same possibility distribution as $\left\{\left(\psi_{j}, \alpha\right): j=1, \ldots \ell\right\}$. Thus, we can still see $B^{N}$ as a conjunction of weighted clauses, i.e., we get an extension of the conjunctive normal form of propositional bases to possibilistic logic.

Moreover, there is another possible understanding of the degree of satisfaction $\pi_{B}^{N}(\omega)$. Consider $B^{N}$ as a fuzzy set of classical formulas, and denote by $\mathcal{N}$, the associated necessity measure on $B^{N}$, defined by $\forall A \subseteq\left\{\varphi_{1}, \ldots, \varphi_{n}\right\}$, $\mathcal{N}(A)=\min _{\varphi_{j} \notin A}\left(1-\alpha_{j}\right)$. For all $j=1, \ldots, n$ we have $\alpha_{j}=1-\mathcal{N}\left(\left\{\varphi_{j}\right\}^{c}\right)$. Note that we do not assume that there exists $i \in\{1, \ldots, m\}$ such that $\alpha_{i}=1$. So the necessity measure defined above is possibly sub-normalized. More precisely, we have $\mathcal{N}(\emptyset)=\min _{i=1}^{m}\left(1-\alpha_{i}\right)$ instead of $\mathcal{N}(\emptyset)=0$. Let $B_{\omega}^{N}=\left\{\varphi:(\varphi, \alpha) \in B^{N}, \omega \models \varphi\right\}$ be the set of logical formulas for which $\omega$ is a model. We have

$$
\pi_{B}(\omega)=\min _{j=1}^{m} \max \left(1-\alpha_{j}, I_{M\left(\varphi_{j}\right)}(\omega)\right)=\min _{\varphi_{j} \notin B_{\omega}}\left(1-\alpha_{j}\right)=\mathcal{N}\left(B_{\omega}^{N}\right) .
$$

\footnotetext{
${ }^{2} N\left(\varphi_{j}\right)$ is short for $N\left(M\left(\varphi_{j}\right)\right)$, i.e. it assigns the same value to semantically equivalent propositions.
} 


\subsubsection{4 -bases}

A dual representation in possibilistic logic is based on guaranteed possibility measures $[7,8]$. We consider another type of logical formula (now denoted between brackets rather than parentheses) as a pair $[\psi, \beta]$, expressing the constraint $\Delta(\psi) \geq \beta$, where $\Delta$ is a guaranteed possibility measure characterized by

$$
\Delta(\phi \vee \psi)=\min (\Delta(\phi), \Delta(\psi))
$$

and $\Delta(\emptyset)=1$. Note that $\Delta$ is decreasing with respect to logical entailment since $\Delta(\phi)=\min _{\omega \models \phi} \pi(\omega)$ (hence one may speak of 'anti-measure' by contrast with the possibility and necessity measures $\Pi$ and $N$ ). In such a context, a base $B^{\Delta}=\left\{\left[\psi_{i}, \beta_{i}\right] \mid i=1, \ldots, n\right\}$ is associated to the distribution

$$
\pi_{B}^{\Delta}(\omega)=\max _{i=1, \ldots, n} \pi_{\left[\psi_{i}, \beta_{i}\right]}(\omega) \quad \text { with } \pi_{\left[\psi_{i}, \beta_{i}\right]}(\omega)= \begin{cases}\beta_{i}, & \text { if } \omega \in M\left(\psi_{i}\right) \\ 0, & \text { otherwise }\end{cases}
$$

So, if the anti-measure $\Delta$ is based on the possibility distribution $\pi$, it holds that $\Delta\left(\psi_{i}\right) \geq \beta_{i}, \forall i=1, \ldots, n$ if and only if $\pi \geq \pi_{B}^{\Delta}$. So, the possibilistic base $B^{\Delta}$ is a description of a possibility distribution "from below".

A dual possibilistic base $B^{\Delta}$ can always be rewritten as a base in which the formulas $\psi_{j}$ are conjunctions of literals (cubes) without altering $\pi_{B}^{A}$, thanks to the decomposability of guaranteed possibility measures with respect to disjunction. So $B^{\Delta}$ can be seen as a weighted combination of cubes, i.e, as an extension of the disjunctive normal form.

A possibilistic logic base $B^{\Delta}$ (a $\Delta$-base for short) expressed in terms of a guaranteed possibility measure can always be rewritten equivalently in terms of a standard possibilistic logic base $B^{N}$ (an $N$-base for short) using necessity measures [1] and conversely, enforcing the equality $\pi_{B}^{N}=\pi_{B}^{A}$. The transformation from $\pi_{B}^{N}$ to $\pi_{B}^{\Delta}$ corresponds to writing the min-max expression of $\pi_{B}^{N}$ as a max-min expression by applying the distributivity of min over max.

Proposition 2. The $N$-base $B^{N}=\left\{\left(\varphi_{j}, \alpha_{j}\right) \mid j=1, \ldots, m\right\}$ is semantically equivalent to the $\Delta$-base $B^{4}=$ $\left\{\left[\wedge_{j \in J} \varphi_{j}, \min _{k \notin J}\left(1-\alpha_{k}\right)\right]: J \subseteq\{1, \ldots, m\}\right\}$.

Proof. Any expression of the form $\min _{j=1}^{m} \max \left(a_{j}, b_{j}\right)$ can be rewritten as $\max _{J \subseteq\{1, \ldots, m\}} \min \left(\min _{j \in J} a_{j}, \min _{j \notin J} b_{j}\right)$, where $\min _{j \in \emptyset} a_{j}=1$. Hence

$$
\begin{aligned}
\pi_{B}^{N}(\omega) & =\min _{j=1}^{m} \max \left(1-\alpha_{j}, I_{M\left(\varphi_{j}\right)}(\omega)\right) \\
& =\max _{J \subseteq\{1, \ldots, m\}} \min \left(\min _{k \notin J}\left(1-\alpha_{k}\right), I_{M\left(\wedge_{j \in J} \varphi_{j}\right)}(\omega)\right)
\end{aligned}
$$

where $I_{M\left(\wedge_{j \in J} \varphi_{j}\right)}(\omega)=\min _{j \in J} I_{M\left(\varphi_{j}\right)}(\omega)$.

Note that it looks as if the translated knowledge base is exponentially larger than the original one. However it can be simplified. Indeed, suppose, without loss of generality that $\alpha_{1}>\alpha_{2}>\cdots>\alpha_{m}$ (recall that if $\alpha_{j}=\alpha_{k}$ it is possible to replace $\left\{\left(\varphi_{j}, \alpha_{j}\right),\left(\varphi_{k}, \alpha_{k}\right)\right\}$ by $\left.\left\{\left(\varphi_{i} \wedge \varphi_{k}, \alpha_{k}\right)\right\}\right)$ and $\alpha_{m+1}=0$ by convention. Then it is easy to check that

$$
\max _{J \subseteq\{1, \ldots, m\}} \min \left(\min _{k \notin J}\left(1-\alpha_{k}\right), I_{M\left(\wedge_{j \in J} \varphi_{j}\right)}(\omega)\right)=\max _{k=1, \ldots, m+1} \min \left(1-\alpha_{k}, I_{M\left(\wedge_{j=1}^{k-1} \varphi_{j}\right)}(\omega)\right)
$$

which corresponds to the $\Delta$-base

$$
B^{4}=\left\{\left[\wedge_{j=1}^{k-1} \varphi_{j}, 1-\alpha_{k}\right]: k=1, \ldots, m+1\right\},
$$

with $\wedge_{j=1}^{0} \varphi_{j}=\top$ (tautology).

Of course, likewise the $\Delta$-base $B^{\Delta}=\left\{\left[\psi_{i}, \beta_{i}\right] \mid i=1, \ldots, n\right\}$ is semantically equivalent to the $N$-base

$$
B^{N}=\left\{\left(\vee_{i \in J} \psi_{i}, \max _{k \notin J}\left(1-\beta_{k}\right)\right): J \subseteq\{1, \ldots, m\}\right\},
$$


which can be simplified as

$$
B^{N}=\left\{\left(\vee_{i=1}^{k-1} \psi_{i}, 1-\beta_{k}\right): k=1, \ldots, n+1\right\},
$$

with $\beta_{1}>\beta_{2}>\cdots>\beta_{m}>\beta_{n+1}=0, \vee_{j=1}^{0} \psi_{j}=\perp$ (contradiction), by convention.

Example 1. $B^{\Delta}=\left\{\left[\psi_{1}, 1\right],\left[\psi_{2}, \beta\right]\right\}$ with $\beta<1$. Let $E_{i}=M\left(\psi_{i}\right)$. We have $\pi(\omega)=\max \left(I_{E_{1}}(\omega), \min \left(I_{E_{2}}(\omega), \beta\right)\right)=$ $\min \left(\max \left(I_{E_{1}}(\omega), I_{E_{2}}(\omega), 0\right), \max \left(I_{E_{1}}(\omega), \beta\right)\right)$. It does correspond to $B^{N}=\left\{\left(\psi_{1} \vee \psi_{2}, 1\right),\left(\psi_{1}, 1-\beta\right)\right\}$.

Note that this procedure generalizes the mutual transformation of a disjunctive normal form into a conjunctive normal form to the gradual case. The existence of equivalent min-max and max-min expressions for the semantics of possibilistic logic bases echoes similar observations that can be made on the equivalent expressions of Sugeno integrals given in Proposition 1.

\section{Fuzzy measures and possibilistic logic bases}

This section presents a method for encoding a fuzzy measure by means of a possibilistic logic base with positive clauses (i.e., clauses with positive literals), and conversely shows that a general possibilistic logic base induces a general set function. Generally, the obtained set function is non-monotonic, but necessary and sufficient conditions are presented for a possibilistic logic base to encode a fuzzy measure. In the decision evaluation context, it means that, in this section, we only consider Boolean criteria that are either satisfied or not. Suppose a language where propositional variables $x_{i} \in\{0,1\}, i=1, \ldots, n$, encode criteria $i \in \mathcal{P}$. An interpretation of $\omega_{x}$ of the language corresponds to a Boolean alternative $x$ for which criteria are fully satisfied or not. For simplicity, we use the same symbol $x$ for the alternative and the corresponding interpretation. As usual $x_{i}$ will denote the case where $x_{i}=1$ and $\neg x_{i}$ the case where $x_{i}=0$. We denote by $T^{x} \subseteq \mathcal{P}$ the set of criteria satisfied by alternative $x$. The subset $T^{x}$ is encoded by the interpretation $\wedge_{i \in T^{x}} x_{i} \wedge \wedge_{i \notin T^{x} \neg x_{i}}$.

\subsection{Fuzzy measures as possibilistic logic bases}

First consider a Boolean fuzzy measure $\mu$ taking values on $\{0,1\}$. For any focal set $T \in \mathcal{F}_{\mu}$, it is clear that $\forall A \subset$ $T, \mu(A)=0$ and $\forall A \supseteq T, \mu(A)=1$. Consider an alternative $x \in\{0,1\}^{n}$. In the decision context, a Boolean fuzzy measure means groups of criteria are either fully important or not at all, so that for an alternative $x$ to be acceptable, it is necessary and sufficient that all criteria in some focal set $T$ of $\mu$ be jointly satisfied, i.e., $\wedge_{i \in T} x_{i}=1$. The logical formula expressing the global acceptability of $x$ is thus $\varphi \equiv \vee_{T \in \mathcal{F}_{\mu}} \wedge_{i \in T} x_{i}$ which is a Boolean polynomial. By construction, no conjunction is redundant in this expression.

Putting this formula in conjunctive normal form, one obtains a logical base of clauses $B_{\mu}$ that exactly describes the priority scheme expressed by the Boolean fuzzy measure, in terms of a conjunction of clauses of the form $\varphi=$ $\vee_{j=1, \ldots, k: i_{j} \in T_{j}} x_{i_{j}}$, one per $k$-tuple of criteria, each criterion $i_{j}$ involved being taken in a different focal element $T_{j} .{ }^{3}$ It is obvious that no such clause contains a negative literal. And we have that $x \vDash B_{\mu}$ if and only if $\mu\left(T^{x}\right)=1$.

Example 2. We consider three criteria $\{1,2,3\}$, the evaluation scale $L=\{0,1\}$ and the following fuzzy measure:

\begin{tabular}{lllllllll}
\hline & $\emptyset$ & $\{1\}$ & $\{2\}$ & $\{3\}$ & $\{1,2\}$ & $\{1,3\}$ & $\{2,3\}$ & $\{1,2,3\}$ \\
\hline$\mu$ & 0 & 0 & 0 & 1 & 1 & 1 & 1 & 1 \\
$\mu_{\#}$ & 0 & 0 & 0 & 1 & 1 & 0 & 0 & 0 \\
\hline
\end{tabular}

We have $\mathcal{F}_{\mu}=\{\{1,2\},\{3\}\}$, which corresponds to the formula $\varphi=\left(x_{1} \wedge x_{2}\right) \vee x_{3}=\left(x_{1} \vee x_{3}\right) \wedge\left(x_{2} \vee x_{3}\right)$. Hence the corresponding possibilistic logic $N$-base is $B=\left\{\left(x_{1} \vee x_{3}, 1\right),\left(x_{2} \vee x_{3}, 1\right)\right\}$.

\footnotetext{
${ }^{3}$ By construction, no such clause is redundant.
} 
Now consider a fuzzy measure $\mu$ taking values on a totally ordered scale $L$. Recalling the fact that its Möbius transform $\mu_{\#}$ is a possibility distribution on $2^{\mathcal{P}}$, the fuzzy measure $\mu$ can be encoded as a $\Delta$-base, interpreting the basic possibility assignment $\mu_{\#}(T)>0$ as the actual possibility degree of the conjunction of atoms corresponding to criteria in $T$.

The $\Delta$-base equivalent to the fuzzy measure $\mu$ is thus

$$
B^{\Delta}(\mu)=\left\{\left[\wedge_{i \in T} x_{i}, \mu_{\#}(T)\right], T \in \mathcal{F}_{\mu}\right\} .
$$

By construction, $\pi_{B^{N}}(x)=\max _{T \in \mathcal{F}_{\mu}} \min \left(\min _{i \in T} x_{i}, \mu_{\#}(T)\right)=\mu\left(T^{x}\right)$, from Proposition 1. It means that $x$ is acceptable at least to degree $\alpha$ if it satisfies all criteria in at least one of the focal sets of $\mu$ of importance level $\alpha$ or greater, i.e. all criteria in some $T$ such that $\mu_{\#}(T) \geq \alpha$.

Using the transformation from the disjunctive to the conjunctive normal form, $B^{\Delta}(\mu)$ can be rewritten as a set $B^{N}(\mu)$ of prioritized positive clauses. To make this transformation, we can proceed by first rank-ordering the subset $\mu_{\#}\left(\mathcal{F}_{\mu}\right)=\left\{\mu_{\#}(T), T \in \mathcal{F}_{\mu}\right\}$ as $\beta_{1}=1>\beta_{2}>\cdots>\beta_{\ell}>0$. Let $\mathcal{F}_{\mu}^{j}=\left\{T, \mu_{\#}(T)=\beta_{j}\right\}$. Then $B^{\Delta}(\mu)=$ $\left\{\left[\vee_{T \in \mathcal{F}_{\mu}^{j}} \wedge_{i \in T} x_{i}, \beta_{j}\right], i=1, \ldots, \ell\right\}$.

Applying the results of Section 2.2, the $N$-base representing the fuzzy measure $\mu$ is (with convention $\beta_{\ell+1}=0$ ):

$$
B^{N}(\mu)=\left\{\left(\vee_{j=1}^{i} \vee_{T \in \mathcal{F}_{\mu}^{j}} \wedge_{k \in T} x_{k}, 1-\beta_{i+1}\right), \quad i=1, \ldots, \ell\right\},
$$

noticing that $\mathcal{N}_{\mu}\left(\left\{\vee_{T \in \mathcal{F}_{\mu}^{j}} \wedge_{i \in T} x_{i}: j=1, \ldots, i\right\}\right)=1-\beta_{i+1}$, where $\mathcal{N}_{\mu}$ is the necessity measure associated to the possibility distribution $\mu_{\#}$ on the power set $2^{\mathcal{P}}$. It is clear that by construction, $\pi_{B^{N}}(x)=\pi_{B^{4}}(x)=\mu\left(T^{x}\right)=\mathcal{N}_{\mu}\left(B_{x}^{N}\right)$, where $B_{x}^{N}=\left\{\varphi_{i}:\left(\varphi_{i}, \gamma_{i}\right) \in B^{N}(\mu), x \in M\left(\varphi_{i}\right)\right\}$. Note that $B_{x}^{N}$ can always be expressed as a set of weighted disjunctions of positive literals.

Example 3. We consider three criteria $\{1,2,3\}$, the importance scale is $L=\left\{0<\lambda_{1}<\lambda_{2}<1\right\}$, where $\lambda_{2}=1-\lambda_{1}$, and the following fuzzy measure:

\begin{tabular}{lllllllll}
\hline & $\emptyset$ & $\{1\}$ & $\{2\}$ & $\{3\}$ & $\{1,2\}$ & $\{1,3\}$ & $\{2,3\}$ & $\{1,2,3\}$ \\
\hline$\mu$ & 0 & $\lambda_{2}$ & $\lambda_{1}$ & 0 & $\lambda_{2}$ & 1 & $\lambda_{2}$ & 1 \\
$\mu_{\#}$ & 0 & $\lambda_{2}$ & $\lambda_{1}$ & 0 & 0 & 1 & $\lambda_{2}$ & 0 \\
\hline
\end{tabular}

Hence we have $\mathcal{F}_{\mu}^{1}=\{\{1,3\}\}, \mathcal{F}_{\mu}^{\lambda_{2}}=\{\{1\},\{2,3\}\}, \mathcal{F}_{\mu}^{\lambda_{1}}=\{\{2\}\}$, hence one directly gets $B^{4}=\left\{\left[p_{1} \wedge p_{3}\right],\left[p_{1} \vee\right.\right.$ $\left.\left.\left(p_{2} \wedge p_{3}\right), \lambda_{2}\right],\left[p_{2}, \lambda_{1}\right]\right\}$. Then, the corresponding possibilistic logic base $B^{N}(\mu)$ is $\left\{\left(p_{1} \wedge p_{3}, 1-\lambda_{2}\right),\left(\left(p_{1} \wedge p_{3}\right) \vee\right.\right.$ $\left.\left.\left.p_{1} \vee\left(p_{2} \wedge p_{3}\right), 1-\lambda_{1}\right),\left(p_{1} \wedge p_{3}\right) \vee p_{1} \vee\left(p_{2} \wedge p_{3}\right) \vee p_{2}, 1\right)\right\}$ and in clausal form, taking subsumption into account: $B^{N}(\mu)=\left\{\left(p_{1}, 1-\lambda_{2}\right),\left(p_{3}, 1-\lambda_{2}\right),\left(p_{1} \vee p_{3}, 1-\lambda_{1}\right),\left(p_{1} \vee p_{2}, 1\right)\right\}{ }^{4}$

As can be observed from the example, the possibilistic logic base $B^{N}(\mu)$ reflects the conjugate $\mu_{c}$ of fuzzy measure $\mu$ defined as $\mu_{c}(A)=1-\mu\left(A^{c}\right)$, where $A^{c}$ denotes the complement of $A$, namely $\mu_{c}(A)=\max _{T_{j} \subseteq A}\{\beta: \varphi=$ $\left.\vee_{j: i \in T_{j}} p_{i},(\varphi, \beta) \in B^{N}(\mu)\right\}$.

Such a representation of a fuzzy measure is unusual in multiple criteria aggregation. However, while the values of the fuzzy measure directly reflect the importance of groups of criteria, we obtain here another view of the interplay between criteria; namely $\left(\varphi_{i}, \alpha_{i}\right) \in B^{N}(\mu)$ means that if all criteria in the disjunction $\varphi_{i}$ are violated, the resulting global evaluation cannot be above $1-\alpha_{i}$.

For instance, the possibilistic base in the above example expresses that violating criteria ' 1 ' and ' 2 ' is forbidden as it leads to the full rejection of the considered alternative. Moreover, violating one of criteria ' 1 ' and ' 3 ' leads to a global evaluation not greater than $\lambda_{2}$, while violating both of them leads to a global evaluation even worse, that is not greater than $\lambda_{1}$, since $0<\lambda_{1}<\lambda_{2}<1$. This result might look surprising since the fuzzy measure value of criterion ' 3 ' is 0 .

\footnotetext{
${ }^{4}$ This base can be more simply written as $\left\{\left(p_{1}, \lambda_{1}\right),\left(p_{3}, \lambda_{1}\right),\left(p_{1} \vee p_{3}, \lambda_{2}\right),\left(p_{1} \vee p_{2}, 1\right)\right\}$, since $\lambda_{1}=1-\lambda_{2}$. But we prefer to keep the notation 1 - so as to lay bare the duality between $\Delta$ - and $N$-bases.
} 
But we should observe that, together, criteria ' 1 ' and ' 3 ' reach importance value 1 , that is, if an alternative satisfies both criteria, it satisfies all formulas in $B^{N}(\mu)$, and gets the maximal global rating.

\subsection{Possibilistic logic bases as set functions}

A set of formulas $\varphi_{j}, j=1, \ldots, m$, in a language where propositional variables $x_{i}$ encode Boolean criteria $i \in \mathcal{P}$ can be viewed as requirements $j \in \mathcal{R}$ expressed as logical combinations of elementary criteria [5]. In such a context, an alternative $x$ satisfies requirement $j$ or not, according to whether it is or not a model of $\varphi_{j}$. So using the characteristic function of $M\left(\varphi_{j}\right)$, the acceptability of an alternative $x$ according to each requirement $j$ can be evaluated by computing $I_{M\left(\varphi_{j}\right)}(x)$.

Now, using the possibility distribution over the models of a possibilistic logic base $B^{N}=\left\{\left(\varphi_{j}, \alpha_{j}\right) \mid j=1, \ldots, m\right\}$ an associated set-function can be defined on $2^{\mathcal{P}}$. More precisely, when we consider a subset $T$ of properties, it can be viewed as a subset $T^{x}$ associated to the Boolean alternative $x^{T}$ with $x_{i}^{T}=1$ if $i \in T$ and 0 otherwise. Hence we can formulate the following definition:

Definition 4. The set function $\mu_{B}$ induced by $B^{N}$ is defined, for all $T \subseteq \mathcal{P}$, by $\mu_{B}(T)=\pi_{B}\left(x^{T}\right)=\wedge_{\varphi_{i} \notin B_{x}^{N}}\left(1-\alpha_{i}\right)$ where $x^{T}=\wedge_{i \in T} x_{i} \wedge \wedge_{i \notin T} \neg x_{i}$, and $B_{x}^{N}=\left\{\varphi_{j}:\left(\varphi_{j}, \alpha_{j}\right) \in B^{N}, x \in M\left(\varphi_{j}\right)\right\}$.

It is clear that since the fuzzy measure is obtained by means of the possibility distribution $\pi_{B}$ induced by $B^{N}$, replacing the latter by its deductive closure is immaterial in the determination of $\mu_{B}$.

Note that if we apply this definition to the $N$-base calculated from a fuzzy measure, it is obvious that we recover this fuzzy measure, that is, $\mu_{B^{N}(\mu)}(T)=\mu(T)$ (they correspond the same possibility distribution on interpretations). To illustrate this point, let us consider the $N$-base obtained in the previous example and let us calculate the associated fuzzy measure.

Example 4. We consider three criteria $\{1,2,3\}$, the scale $L=\left\{0<\lambda_{1}<\lambda_{2}<1\right\}$, where $\lambda_{2}=1-\lambda_{1}$, and the possibilistic logic base $B^{N}=\left\{\left(x_{1}, 1-\lambda_{2}\right),\left(x_{3}, 1-\lambda_{2}\right),\left(x_{1} \vee x_{3}, 1-\lambda_{1}\right),\left(x_{1} \vee x_{2}, 1\right)\right\}$. Let $\varphi_{1}=x_{1}, \varphi_{2}=x_{3}, \varphi_{3}=$ $x_{1} \vee x_{3}$ and $\varphi_{4}=x_{1} \vee x_{2}$. Let us calculate the associated fuzzy measure for all the subsets of criteria.

\begin{tabular}{llll}
\hline The set A & The associated alternative $\mathrm{x}$ & $B_{x}^{N}$ & $\mu(A)=\mathcal{N}\left(B_{x}^{N}\right)$ \\
\hline$\{1,2,3\}$ & $(1,1,1)$ & $\left\{\varphi_{1}, \varphi_{2}, \varphi_{3}, \varphi_{4}\right\}$ & 1 \\
$\{1,2\}$ & $(1,1,0)$ & $\left\{\varphi_{1}, \varphi_{3}, \varphi_{4}\right\}$ & $\lambda_{2}$ \\
$\{1,3\}$ & $(1,0,1)$ & $\left\{\varphi_{1}, \varphi_{2}, \varphi_{3}, \varphi_{4}\right\}$ & 1 \\
$\{2,3\}$ & $(0,1,1)$ & $\left\{\varphi_{2}, \varphi_{3}, \varphi_{4}\right\}$ & $\lambda_{2}$ \\
$\{1\}$ & $(1,0,0)$ & $\left\{\varphi_{1}, \varphi_{3}, \varphi_{4}\right\}$ & $\lambda_{2}$ \\
$\{2\}$ & $(0,1,0)$ & $\left\{\varphi_{4}\right\}$ & $\lambda_{1}$ \\
$\{3\}$ & $(1,1,1)$ & $\left\{\varphi_{2}, \varphi_{3}\right\}$ & 0 \\
\hline
\end{tabular}

The obtained fuzzy measure is the fuzzy measure used in the previous example.

While the $N$-base obtained from a fuzzy measure contains only positive clauses, the set function $\mu_{B}$ obtained from any possibilistic logic base $B^{N}$ is not always a fuzzy measure since some clauses $\varphi_{j}$ in an $N$-base may contain negated terms. This feature accounts for a kind of negative synergy between properties, that can be expressed using a possibilistic logic description of acceptability requirements.

Example 5. We consider three properties $\{1,2,3\}$, the scale $L=\left\{0<\lambda_{1}<1\right\}$, where $\lambda_{1}=1-\lambda_{1}$, and the $N$-base $B=\left\{\left(x_{1}, 1\right),\left(x_{2} \vee x_{3}, 1\right),\left(x_{2}, 1-\lambda_{1}\right),\left(\neg x_{2} \vee \neg x_{3}, 1\right)\right\}$.

Let $\varphi_{1}=x_{1}, \varphi_{2}=x_{2} \vee x_{3}, \varphi_{3}=x_{2}$ and $\varphi_{4}=\neg x_{2} \vee \neg x_{3}$. It means that it is imperative to satisfy criterion 1 , but criteria 2 and 3 must be neither both satisfied nor both violated, and violating criterion 2 leads to a global evaluation not greater than $1-\lambda_{1}$. 
Let us calculate the set-function $\mu$. The reader can check it is not a fuzzy measure.

\begin{tabular}{llll}
\hline$T^{x}$ & $x$ & $B_{x}^{N}$ & $\mu\left(T^{x}\right)=\pi_{B}(x)=\mathcal{N}\left(B_{x}^{N}\right)$ \\
\hline$\{1,2,3\}$ & $(1,1,1)$ & $\left\{\varphi_{1}, \varphi_{2}, \varphi_{3}\right\}$ & 0 \\
$\{1,2\}$ & $(1,1,0)$ & $\left\{\varphi_{1}, \varphi_{2}, \varphi_{3}, \varphi_{4}\right\}$ & 1 \\
$\{1,3\}$ & $(1,0,1)$ & $\left\{\varphi_{1}, \varphi_{2}, \varphi_{4}\right\}$ & $\lambda_{1}$ \\
$\{2,3\}$ & $(0,1,1)$ & $\left\{\varphi_{2}, \varphi_{3}\right\}$ & 0 \\
$\{1\}$ & $(1,0,0)$ & $\left\{\varphi_{1}, \varphi_{4}\right\}$ & 0 \\
$\{2\}$ & $(0,1,0)$ & $\left\{\varphi_{2}, \varphi_{3}, \varphi_{4}\right\}$ & 0 \\
$\{3\}$ & $(1,1,1)$ & $\left\{\varphi_{2}, \varphi_{4}\right\}$ & 0 \\
\hline
\end{tabular}

In order to obtain a fuzzy measure, the set function $\mu_{B}$ has to satisfy the properties $\mu_{B}(\emptyset)=0, \mu_{B}(\mathcal{P})=1$ and $S \subseteq T$ entails $\mu_{B}(S) \leq \mu_{B}(T)$. When we consider a Boolean alternative $x, \varphi$ does not follow from $B_{x}^{N}$ whenever $\varphi$ has the form $\vee_{i \in I} \neg x_{i} \vee \vee_{j \in J} x_{j}$ where $I \subseteq T^{x}$ and $J \subseteq T^{x c}$ where $T^{x c}$ is the complement of $T^{x}$. So, as $\mathcal{N}\left(B_{x}^{N}\right)=\wedge_{\varphi_{i} \notin B_{x}^{N}}\left(1-\alpha_{i}\right)=\mu_{B}\left(T^{x}\right)$ we can identify the properties a possibilistic logic base needs to have in order to be associated to a fuzzy measure.

Proposition 3. The following equivalences hold, given an $N$-base $B^{N}$ in clausal form:

- $\mu_{B}(\mathcal{P})=1$ if and only if $B^{N} \not \neq\left(\vee_{i \in I} \neg x_{i}, \alpha\right), \forall I \subseteq\{1, \ldots, m\}, \forall \alpha>0$.

- $\mu_{B}(\emptyset)=0$ if and only if there exists a subset $J$ of $\{1, \ldots, m\}$ and a clause $\varphi=\vee_{j \in J} x_{j}$ such that $B^{N} \models(\varphi, 1)$.

- $\forall S \subseteq T, \mu_{B}(S) \leq \mu_{B}(T)$ if and only if $\forall S \subseteq T, \max _{\varphi_{j} \notin B_{x}^{N} T} \alpha_{j} \leq \max _{\varphi_{i} \notin B_{x}^{N} S} \alpha_{i}$.

Proof. The first equivalence: $x^{\mathcal{P}}=(1,1, \ldots, 1)=\wedge_{i \in \mathcal{P}} x_{i} . \mu_{B}(\mathcal{P})=1$ if and only if $x^{\mathcal{P}}$ satisfies all formulas in $B^{N}$. But the clauses violated by $x^{\mathcal{P}}$ are all of the form $\vee_{i \in I} \neg x_{i}$ for some non-empty set $I$ of variables. Hence the result.

The second equivalence: $x^{\emptyset}=(0,0, \ldots, 0)=\wedge_{i \in \mathcal{P}} \neg x_{i} \cdot \mu_{B}(\emptyset)=0$ if and only if $x^{\emptyset}$ violates at least one formula of priority 1 in $B^{N}$. But the clauses violated by $x^{\emptyset}$ are all of the form $\vee_{i \in I} x_{i}$ for some non-empty set $I$ of variables. So $\mu_{B}(\emptyset)=0$ if and only if $B^{N}$ contains at least one such formula with priority 1 .

The third equivalence: $\forall S \subseteq T \in \mathcal{P}, \mu_{B}(S) \leq \mu_{B}(T)$ if and only if $\forall S \subseteq T, \mathcal{N}\left(B_{x}^{N}\right) \leq \mathcal{N}\left(B_{x^{T}}^{N}\right)$. The latter reads $\wedge_{\varphi_{i} \notin B_{x^{S}}^{N}}\left(1-\alpha_{i}\right) \leq \wedge_{\varphi_{i} \notin B_{x^{T}}^{N}}\left(1-\alpha_{i}\right)$ which is equivalent to $\vee_{\varphi_{i} \notin B_{x^{T}}^{N}} \alpha_{i} \leq \vee_{\varphi_{i} \notin B_{x^{S}}^{N}} \alpha_{i}$.

Example 6. First let us consider the possibilistic logic base of Example 4: $B^{N}=\left\{\left(x_{1}, 1-\lambda_{2}\right),\left(x_{3}, 1-\lambda_{2}\right),\left(x_{1} \vee x_{3}\right.\right.$, $\left.\left.1-\lambda_{1}\right),\left(x_{1} \vee x_{2}, 1\right)\right\}$.

- There are no formulas with negation so $\mu(\mathcal{P})=1$.

- The logical formula $\left(x_{1} \vee x_{2}, 1\right)$ entails $\mu(\emptyset)=0$.

- $\{1\} \subseteq\{1,2\}: \vee_{\left.\varphi_{i} \notin B_{x^{1}}^{N}, 2\right\}} \alpha_{i}=1-\lambda_{2}=\vee_{\varphi_{i} \notin B_{x^{11}}^{N}}$;

$\{1\} \subseteq\{1,3\}: \vee_{\varphi_{i} \notin B_{x\{1,3\}}^{N}} \alpha_{i}=0 \leq \vee_{\varphi_{i} \notin B_{x^{\prime 11}}^{N}} \alpha_{i}=1-\lambda_{2} ;$

$\{3\} \subseteq\{1,3\}: \vee_{\varphi_{i} \notin B_{x^{\{1,3\}}}^{N}} \alpha_{i}=0 \leq \vee_{\varphi_{i} \notin B_{x^{\{1\}}}^{N}} \alpha_{i}=1$.

So the possibilistic logic base is associated to a fuzzy measure.

Next, consider the possibilistic logic base of Example 5: $B^{N}=\left\{\left(x_{1}, 1\right),\left(x_{2} \vee x_{3}, 1\right),\left(x_{2}, 1-\lambda_{2}\right),\left(\neg x_{2} \vee \neg x_{3}, 1\right)\right\}$. We have $\vee_{\varphi_{i} \notin B_{x}^{N}{ }^{11,2,3\}}} \alpha_{i}=1>\vee_{\varphi_{i} \notin B_{x^{\prime 1,2\}}}^{N}} \alpha_{i}=0$ so $B$ is not associated to a fuzzy measure.

The question raised by Proposition 3 has to do with monotone Boolean functions. First, in the classical case, a set of requirements encoded as a standard propositional knowledge base $B$ is equivalent to a Boolean function whose arguments are Boolean attributes $x_{i}$ taking values 0 or 1 according to whether the alternative is acceptable or not. The (increasing) monotonicity of the corresponding set-function $\mu_{B}$ is equivalent to the monotonicity of the Boolean function. And it is known that a Boolean function is monotone if and only its reduced disjunctive normal form contains only 0,1 and positive literals. It corresponds to the associated $\Delta$-base. The conjunctions of literals in this form here correspond to focal elements of $\mu_{B}$. 
In the more general case, the third point of Proposition 3 is not easy to interpret. However, based on the above results for the Boolean case, one may say that the monotonicity of the set-function $\mu_{B}$ induced by the $N$-base $B^{N}$ is easier to check on the equivalent $\Delta$-base $B^{4}$. Let $\beta_{1}>\beta_{2}>\cdots>\beta_{k}$ be the distinct weights appearing in $B^{4}$, and $B_{i}{ }_{i}$ be the $\Delta$-base containing all terms with weight $\beta_{i}$. Then $\mu_{B}$ will be monotone if and only if all Boolean functions associated to the subbases $B_{i}^{\Delta}$ are monotone, that is, if each $B_{i}^{\Delta}$, put in non-redundant form, contains only conjunctions of positive literals (corresponding to the qualitative Möbius transform of $\mu_{B}$ ). In other words, an $N$-base $B^{N}$ induces a monotonic set-function if and only if it is logically equivalent to another $N$-base where no negative literal appears. While this formulation is easier to understand than the third condition of Proposition 3, the latter is easier to automatically check in practice.

\section{Sugeno integrals and possibilistic logic bases}

Let $\oint_{\mu}(x)$ be a Sugeno integral based on a fuzzy measure $\mu$ and a tuple $x$ of local evaluations which both take their values in a finite totally ordered scale $L$. In the previous section, assuming $x$ is Boolean, we have shown how to compute $\oint_{\mu}(x)$ as the degree of satisfaction of a possibilistic knowledge base by an interpretation of the language generated by the Boolean attributes $x_{i}$. In that case, $\oint_{\mu}(x)=\mu\left(T^{x}\right)$ and the Sugeno integral coincides with a fuzzy measure.

In this section we are going to generalize the previous results concerning fuzzy measures to Sugeno integrals when attributes are no longer Boolean. More precisely, we are going to present how to encode a Sugeno integral by means of a possibilistic logic base and how some possibilistic logic bases can be interpreted as a Sugeno integral.

\subsection{From Sugeno integrals to possibilistic logic bases}

The starting point is to turn a Sugeno integral into a $\Delta$-base, based on the fact that the expression of the former in terms of the qualitative Möbius transform $\oint_{\mu}(x)=\max _{T \subseteq \mathcal{F}_{\mu}} \min \left(\mu_{\#}(T), \min _{i \in T} x_{i}\right)$ is similar to the expression of the possibility distribution of a $\Delta$-base $B^{\Delta}(\mu)=\left\{\left[\wedge_{j \in T} \psi_{j}, \mu_{\#}(T)\right]: T \in \mathcal{F}_{\mu}\right\}$, except that in the latter, $\psi_{j}$ is Boolean, while in $\min _{i \in T} x_{i}, x_{i} \in L$. So we must express an alternative $x$ in terms of weighted Boolean alternatives.

For each criterion $i$, we define $|L|-1$ predicates $p_{i}(\gamma)$, where $\gamma \neq 0 \in L$ with the following convention: $p_{i}(\gamma)$ is true if and only if $x_{i} \geq \gamma$. In the previous section, where $x$ is Boolean, $p_{i}(1)$ was simply denoted by the atom $x_{i}$. Note that we can restrict the scope of $p_{i}(\gamma)$ to $\gamma>0$ since, under our convention, $p_{i}(0)$ is a tautology (and thus is not equivalent to $\neg x_{i}$ in the previous section). We thus consider a simple first order language $\mathcal{L}$ with atoms $\left\{p_{i}(\gamma): i=1, \ldots, n ; \gamma>0 \in L\right\}$, and connectives of negation, conjunction and disjunction. Moreover, we have the following set of domain axioms:

$$
\vdash p_{i}(\gamma) \vee \neg p_{i}(\delta), \quad \forall \delta>\gamma>0, i=1, \ldots, n .
$$

This language will be interpreted in the set of alternatives $x \in L^{\mathcal{P}}$. We define the satisfaction relation as follows:

- $x \vDash p_{i}(\gamma)$ if and only if $x_{i} \geq \gamma$;

- $x \models \neg p_{i}(\gamma)$ if and only if $x_{i}<\gamma$;

- $x \vDash \varphi \wedge \psi$ if and only if $x \vDash \varphi$ and $x \vDash \psi$;

- $x \vDash \varphi \vee \psi$ if and only if $x \vDash \varphi$ or $x \vDash \psi$.

An alternative $x=\left(x_{1}, \ldots, x_{n}\right)$ clearly satisfies the formula $\wedge_{i=1}^{n} p_{i}\left(x_{i}\right)$, and can be viewed as the Boolean interpretation $\wedge_{i=1}^{n}\left(\wedge_{\gamma \leq x_{i}} p_{i}(\gamma)\right) \wedge\left(\wedge_{\gamma>x_{i}} \neg p_{i}(\gamma)\right)$. A logical rendering of the Sugeno integral of $x$ with respect to a fuzzy measure $\mu$ will rely on the use of $\Delta$-possibilistic logic formulas as follows. Each term $\min \left(\mu_{\#}(T), \min _{i \in T} x_{i}\right)$ is encoded by the $\Delta$-base

$$
B_{T}^{\Delta}(\mu)=\left\{\left[\wedge_{i \in T} p_{i}(\gamma), \gamma\right]: \gamma \leq \mu_{\#}(T)\right\}
$$

Lemma 1. $\pi_{B_{T}^{A}(\mu)}(x)=\min \left(\mu_{\#}(T), \min _{i \in T} x_{i}\right)$

Proof. $\pi_{B_{T}^{4}(\mu)}(x)=\max _{\gamma \leq \mu_{\#}(T)} \min \left(\gamma, \min _{i \in T} I_{M\left(p_{i}(\gamma)\right)}(x)\right)$. 
Note that $I_{M\left(p_{i}(\gamma)\right)}(x)=0$ as soon as $\gamma>x_{i}$, and the term $\min \left(\gamma, I_{M\left(p_{i}(\gamma)\right)}(x)\right)$ is subsumed by (i.e., less than) $\min \left(x_{i}, I_{M\left(p_{i}\left(x_{i}\right)\right)}(x)\right)=x_{i}$ if $\gamma \leq x_{i}$. Now,

- either $\mu_{\#}(T) \leq x_{i}, \forall i \in T$ and then $\left.I_{M\left(p_{i}\left(\mu_{\#}(T)\right)\right)}(x)\right)=1, \forall i \in T$, hence $\pi_{B_{T}^{A}(\mu)}(x)=\mu_{\#}(T)$;

- or $\mu_{\#}(T)>\min _{i \in T} x_{i}$, and then $\left.\min _{i \in T} I_{M\left(p_{i}(\gamma)\right)}(x)\right)=0, \forall \gamma$ such that $\min _{i \in T} x_{i}<\gamma \leq \mu_{\#}(T)$, hence $\pi_{B_{T}^{A}(\mu)}(x)=$ $\min _{i \in T} x_{i}$.

Then we easily derive the following result, making the union of the bases $B_{T}^{\Delta}(\mu)$, one for each focal set $T$ in $\mathcal{F}_{\mu}$ :

Proposition 4. The $\Delta$-base $B^{\Delta}(\mu)=\bigcup_{T \in \mathcal{F}_{\mu}} B_{T}^{\Delta}(\mu)$ encodes the Sugeno integral $\oint_{\mu}(x)$, that is: $\oint_{\mu}(x)=\pi_{B^{\Delta}(\mu)}(x)$.

Proof. This is obvious since $\pi_{B_{1} \cup B_{2}}=\max \left(\pi_{B_{1}}, \pi_{B_{2}}\right)$ for $\Delta$-bases.

However, $B^{\Delta}(\mu)$ is large and contains redundant formulas. Instead of building one base per focal set, one may proceed levelwise, building one base per value in the scale $L$, and considering the inequalities $\oint_{\mu}(x) \geq \lambda_{i}$ for each $\lambda_{i} \in L$. Let us begin with a formal characterization of tuples $x$ that satisfy this inequality.

Proposition 5. $\oint_{\mu}(x) \geq \gamma$ if and only if $\exists T \in \mathcal{F}_{\mu}$ s.t. $\mu_{\#}(T) \geq \gamma$ and $\forall i \in T, x_{i} \geq \gamma$.

Proof. Let us use again the identity $\oint_{\mu}(x)=\max _{T \in \mathcal{F}_{\mu}} \min \left(\mu_{\#}(T), \min _{i \in T} x_{i}\right)$. Then clearly, $\oint_{\mu}(x) \geq \gamma$ if and only if $\exists T \in \mathcal{F}_{\mu}, \min \left(\mu_{\#}(T), \min _{i \in T} x_{i}\right) \geq \gamma$. For such a focal set $T$, the condition $\mu_{\#}(T) \geq \gamma$ must hold, as well as $\min _{i \in T} x_{i} \geq \gamma$, i.e. $\forall i \in T, x_{i} \geq \gamma$ must be satisfied.

This easy result says that $x$ is evaluated at least at level $\gamma$ if and only if it satisfies to degree at least $\gamma$ all criteria of a focal set having importance at least $\gamma$. In fact, it is clear that we only need the smallest focal elements in the sense of inclusion among those such that $\mu_{\#}(T) \geq \gamma$. Denote them by $\mathcal{F}_{\mu}^{\gamma}=\min _{\subseteq}\left\{T: \mu_{\#}(T) \geq \gamma\right\}$ (they are the focal sets of the Boolean set function $\mu^{\gamma}(T)=1$ if and only if $\left.\mu(T) \geq \gamma\right)$. Then

Proposition 6. $\oint_{\mu}(x) \geq \gamma$ if and only if $\exists T \in \mathcal{F}_{\mu}^{\gamma}, \forall i \in T, x_{i} \geq \gamma$.

We can then characterize the set of objects $x$ such that $\oint_{\mu}(x) \geq \gamma$, as the set of interpretations of

$$
B_{\gamma}^{\Lambda}(\mu)=\left\{\left[\wedge_{i \in T} p_{i}(\gamma), 1\right]: T \in \mathcal{F}_{\mu}^{\gamma}\right\}
$$

Note that this base does not contain formulas $\left[\wedge_{i \in T} p_{i}(\gamma), 1\right], T \in \mathcal{F}_{\mu}$ for which $\exists S \subset T$ such that $S \in \mathcal{F}_{\mu}$ as per Proposition 6, i.e., it contains no redundant formula. $B_{\gamma}^{\Delta}(\mu)$ is actually the Boolean formula $\vee_{T \in \mathcal{F}_{\mu}^{\gamma}} \wedge_{j \in T} p_{j}(\gamma)$.

Proposition 7. $\oint_{\mu}(x) \geq \gamma$ if and only if $\pi_{B_{\gamma}^{\perp}(\mu)}(x)=1$.

Proof. $\oint_{\mu}(x) \geq \gamma$ if and only if $\exists T, \mu_{\#}(T) \geq \gamma$ and $\forall i \in T, x_{i} \geq \gamma$ if and only if $\exists T, \mu_{\#}(T) \geq \gamma$ such that $\wedge_{i \in T} p_{i}(\gamma)$ holds, if and only if $\pi_{B_{\gamma}{ }^{4}(\mu)}(x)=1$.

The $\Delta$-base $B^{\Delta}(\mu)$, constructed from each focal set of $\mu$ is logically equivalent to the union $B_{\uparrow}^{\Delta}(\mu)=\left\{\left[\wedge_{j \in T} p_{j}(\gamma), \gamma\right]\right.$ : $\left.\forall T \in \mathcal{F}_{\mu}^{\gamma}, \forall \gamma>0 \in L\right\}$ of all $B_{\gamma}^{\perp}(\mu)$ after formulas are assigned weight $\gamma \cdot B_{\uparrow}^{\Lambda}(\mu)$ is obtained from $B^{\Delta}(\mu)$ after removing the redundant weighted clauses in the latter. The main result of this section then follows easily:

Proposition 8. $\pi_{B_{\uparrow}^{A}(\mu)}(x)=\pi_{B^{4}(\mu)}(x)=\oint_{\mu}(x)$.

Proof. $\oint_{\mu}(x)=\max _{\gamma \in L} \min \left(\gamma, I_{\oint_{\mu}(x) \geq \gamma}(x)\right)=\max _{\gamma \in L} \min \left(\gamma, \pi_{B_{\gamma}^{A}(\mu)}(x)\right)$, due to Proposition 7, which is $\pi_{B_{\uparrow}^{A}(\mu)}(x)$. Then use Proposition 4. 
The $\Delta$-base $B_{\uparrow}^{A}(\mu)$ is not redundant by construction. Indeed, if it were, there would be two cubes in it of the form $\left[\wedge_{j \in T} p_{j}(\gamma), \gamma\right],\left[\wedge_{j \in S} p_{j}(\delta), \delta\right]$ such that $T \subseteq S$ and $\delta \leq \gamma$, where $S \in \mathcal{F}_{\delta}, T \in \mathcal{F}_{\gamma}$. But this is impossible as, if $\delta=\gamma$ and $T \neq S$, then $S$ cannot be a focal set of $\mu_{\gamma}$, and $\delta<\gamma$ is incompatible with $T \subseteq S$.

The minimal $\Delta$-base $B_{\uparrow}^{A}(\mu)$ can be turned into an $N$-base if needed, using the technique presented in Section 2 . This transformation is clearly equivalent to the change from a max - min expression of Sugeno integral to a min $-\max$ expression as appears in Proposition 1. Note that in case of binary alternatives, identifying $p_{i}(1)$ to atoms $x_{i}$ we recover the $\Delta$-possibilistic base $B^{\Delta}(\mu)$ identified in the previous section (Eq. (1)).

Example 7. We consider three criteria $\mathcal{P}=\{1,2,3\}$ and the evaluation scale $L=\left\{1, \lambda_{2}, \lambda_{1}, 0\right\}$ with $1>\lambda_{2}>\lambda_{1}>$ 0 . We consider a Sugeno integral with respect to $\mu$ defined by $\mu(\{1\})=\mu(\{2\})=\lambda_{1}, \mu(\{3\})=\lambda_{2}, \mu(\{1,2\})=\lambda_{1}$, $\mu(\{2,3\})=\lambda_{2}, \mu(\{1,3\})=\mu(\{1,2,3\})=1$.

$\mu$ is associated to the following qualitative Möbius transform:

$\mu_{\#}(\{1\})=\mu_{\#}(\{2\})=\lambda_{1}, \mu_{\#}(\{3\})=\lambda_{2}$ and $\mu_{\#}(\{1,3\})=1$.

1. Proceeding by focal sets: $B_{\{1\}}^{\Delta}(\mu)=\left\{\left[p_{1}\left(\lambda_{1}\right), \lambda_{1}\right]\right\}, B_{\{2\}}^{\Delta}(\mu)=\left\{\left[p_{2}\left(\lambda_{1}\right), \lambda_{1}\right]\right\}, B_{\{3\}}^{\Delta}(\mu)=\left\{\left[p_{3}\left(\lambda_{1}\right), \lambda_{1}\right]\right.$, $\left.\left[p_{3}\left(\lambda_{2}\right), \lambda_{2}\right]\right\}$,

$B_{\{1,3\}}^{\Lambda}(\mu)=\left\{\left[p_{1}(1) \wedge p_{3}(1), 1\right],\left[p_{1}\left(\lambda_{2}\right) \wedge p_{3}\left(\lambda_{2}\right), \lambda_{2}\right],\left[p_{1}\left(\lambda_{1}\right) \wedge p_{3}\left(\lambda_{1}\right), \lambda_{1}\right]\right\}$.

$B^{\Delta}(\mu)$ this contains 7 cubes, but $\left[p_{1}\left(\lambda_{2}\right) \wedge p_{3}\left(\lambda_{2}\right), \lambda_{2}\right]$ is subsumed by $\left[p_{3}\left(\lambda_{2}\right), \lambda_{2}\right]$, and $\left[p_{1}\left(\lambda_{1}\right) \wedge p_{3}\left(\lambda_{1}\right), \lambda_{1}\right]$ by $\left[p_{1}\left(\lambda_{1}\right), \lambda_{1}\right]$.

2. Proceeding by levels:

- We want to find $x$ such that $\oint_{\mu}(x) \geq \lambda_{1}$. It is clear that $\mathcal{F}_{\mu}^{\lambda_{1}}=\{\{1\},\{2\},\{3\}\}$. So $B_{\lambda_{1}}^{\Lambda}(\mu)=\left\{\left[p_{1}\left(\lambda_{1}\right), 1\right],\left[p_{3}\left(\lambda_{1}\right), 1\right]\right.$ $\left.\left[p_{2}\left(\lambda_{1}\right), 1\right]\right\}$. So $x$ satisfies $\oint_{\mu}(x) \geq \lambda_{1}$ provided that $x_{1} \geq \lambda_{1}$ or $x_{3} \geq \lambda_{1}$ or $x_{2} \geq \lambda_{1}$, i.e. for any $x$ greater than $\left(\lambda_{1}, 0,0\right)$ or $\left(0, \lambda_{1}, 0\right)$ or $\left(0,0, \lambda_{1}\right)$.

- We want to find $x$ such that $\oint_{\mu}(x) \geq \lambda_{2}$. It is clear that $\mathcal{F}_{\mu}^{\lambda_{2}}=\{\{3\}\}$. So $B_{\lambda_{1}}^{4}(\mu)=\left\{\left[p_{3}\left(\lambda_{2}\right), 1\right]\right\}$. So $x$ satisfies $\oint_{\mu}(x) \geq \lambda_{2}$ provided that $x_{3} \geq \lambda_{2}$, i.e., for any $x$ greater than $\left(0,0, \lambda_{2}\right)$ satisfies $\oint_{\mu}(x) \geq \lambda_{2}$.

- We want to find the alternatives $x$ such that $\oint_{\mu}(x)=1$. It is clear that $\mathcal{F}_{\mu}^{1}=\{\{1,3\}\}$ and $B_{1}^{\Delta}=\left\{\left[p_{1}(1) \wedge p_{3}(1), 1\right]\right.$, so all $x$ greater than $(1,0,1)$ satisfy $\oint_{\mu}(x)=1$.

It corresponds to the following $\Delta$-possibilistic logic base:

$B_{\uparrow}^{\Lambda}(\mu)=\left\{\left[p_{1}(1) \wedge p_{3}(1), 1\right],\left[p_{3}\left(\lambda_{2}\right), \lambda_{2}\right],\left[p_{3}\left(\lambda_{1}\right), \lambda_{1}\right],\left[p_{1}\left(\lambda_{1}\right), \lambda_{1}\right],\left[p_{2}\left(\lambda_{1}\right), \lambda_{1}\right]\right\}$.

It is the one we obtained after deleting redundant cubes in $B^{4}(\mu)$.

So, the Sugeno integral can be interpreted as follows:

- An object that satisfies properties 1 and 3 to degree 1 is fully satisfactory.

- An object that satisfies property 3 to at least level $\lambda_{2}$ is satisfactory at least to degree $\lambda_{2}$.

- An object that satisfies any of properties $1-3$ to degree $\lambda_{1}$ is satisfactory at least to degree $\lambda_{1}$.

\subsection{From $\Delta$-bases to Sugeno integrals}

Consider a $\Delta$-possibilistic base similar to the one obtained in the previous subsection, $B^{4}=\left\{\left[\wedge_{j \in T_{i}} p_{j}\left(\beta_{i}\right), \beta_{i}\right]\right.$, $\left.T_{i} \subseteq \mathcal{P}, \beta_{i}>0 \in L, i=1, \ldots, k\right\}$ we want to identify the class of fuzzy measures $\mu$ such that $\pi_{B^{\Delta}}(x)=$ $\oint_{\mu}(x)$.

First we try to find the class of fuzzy measures induced by one logical formula $\left[\wedge_{j \in T} p_{j}(\beta), \beta\right]$. It is clear that $\forall x, \pi_{\left[\wedge_{j \in T} p_{j}(\beta), \beta\right]}(x) \leq \beta$. Hence we just need to identify the fuzzy measures $\mu$ such that $\pi_{\left[\wedge_{j \in T} p_{j}(\beta), \beta\right]}(x) \geq \beta$. In order to calculate these solutions, let us make a brief reminder of the result proved in [27].

We consider the equation $\oint_{\mu}(x) \geq \beta$ for a fixed $x$. First we denote by $A_{x, \beta}^{\geq}$the set of criteria whose evaluation in $x$ is greater than or equal to $\beta$ and the following fuzzy measure:

$$
\forall A \subseteq \mathcal{P}, \quad \check{\mu}^{x, \beta}(A)=\left\{\begin{array}{ll}
\beta & \text { if } A_{x, \beta}^{\geq} \subseteq A, \\
0 & \text { otherwise },
\end{array} \check{\mu}^{x, \beta}(\emptyset)=0, \quad \check{\mu}^{x, \beta}(\mathcal{P})=1 .\right.
$$


Hence the following result can be proved [27]:

\section{Proposition 9.}

$$
\left\{\mu: \oint_{\mu}(x) \geq \beta\right\}=\left\{\begin{array}{l}
\varnothing \quad \text { if } \max _{i \in T} x_{i}<\beta \\
\left\{\mu: \check{\mu}^{x, \beta} \leq \mu\right\} \text { otherwise }
\end{array}\right.
$$

In our context we consider $k$ inequalities induced by the $\Delta$-bases of the form $\oint_{\mu}\left(x^{i}\right) \geq \beta_{i}$, where $x_{j}^{i}=\beta_{i}$ if $j \in T_{i}$ and 0 otherwise. The set of solutions is $\left\{\mu: \mu \geq \vee_{i=1}^{k} \check{\mu}^{x^{i}, \beta_{i}}\right\}$.

Example 8. Let us consider the following possibilistic logic base and let us calculate the associated Sugeno integral:

$$
B_{\mu}^{\Lambda}=\left\{\left[p_{1}(1) \wedge p_{3}(1), 1\right],\left[p_{3}\left(\lambda_{2}\right), \lambda_{2}\right],\left[p_{1}\left(\lambda_{1}\right), \lambda_{1}\right],\left[p_{2}\left(\lambda_{1}\right), \lambda_{1}\right],\left[p_{3}\left(\lambda_{1}\right), \lambda_{1}\right]\right\} .
$$

We want to find $\mu$ such that $\oint_{\mu}\left(0,0, \lambda_{1}\right) \geq \lambda_{1}, \oint_{\mu}\left(0, \lambda_{1}, 0\right) \geq \lambda_{1}, \oint_{\mu}\left(\lambda_{1}, 0,0\right) \geq \lambda_{1}, \oint_{\mu}\left(0,0, \lambda_{2}\right) \geq \lambda_{2}$ and $\oint_{\mu}(1,0,1) \geq 1$. We obtain the following fuzzy measures: $\forall A \subset \mathcal{P}$,

$$
\begin{aligned}
& \mu_{1}=\check{\mu}^{\left(0,0, \lambda_{1}\right), \lambda_{1}}(A)=\lambda_{1} \text { if }\{3\} \subseteq A, \text { and } 0 \text { otherwise; } \\
& \mu_{2}=\check{\mu}^{\left(0, \lambda_{1}, 0\right), \lambda_{1}}(A)=\lambda_{1} \text { if }\{2\} \subseteq A, \text { and } 0 \text { otherwise; } \\
& \mu_{3}=\check{\mu}^{\left(\lambda_{1}, 0,0\right), \lambda_{1}}(A)=\lambda_{1} \text { if }\{1\} \subseteq A, \text { and } 0 \text { otherwise; } \\
& \mu_{4}=\check{\mu}^{\left(0,0, \lambda_{2}\right), \lambda_{2}}(A)=\lambda_{2} \text { if }\{3\} \subseteq A, \text { and } 0 \text { otherwise; } \\
& \mu_{5}=\check{\mu}^{(1,0,1), 1}(A)=1 \text { if }\{1,3\} \subseteq A, \text { and } 0 \text { otherwise. }
\end{aligned}
$$

So the solutions are fuzzy measures greater than $\vee_{i=1}^{5} \mu_{i}$ whose values are given in the following table:

\begin{tabular}{lllllll}
\hline & $\{1\}$ & $\{2\}$ & $\{3\}$ & $\{1,2\}$ & $\{1,3\}$ & $\{2,3\}$ \\
\hline$\vee_{i=1}^{5} \mu_{i}$ & $\lambda_{1}$ & $\lambda_{1}$ & $\lambda_{2}$ & $\lambda_{1}$ & 1 & $\lambda_{2}$ \\
\hline
\end{tabular}

We retrieve the fuzzy measure we started with in the previous example.

Note that if we start from any $\Delta$-base, we just need to

- put $B^{\Delta}$ in disjunctive normal form, that is, put each formula $\phi$ containing only positive literals of the form $p_{i}(\beta)$, such that $[\phi, \beta] \in B^{\Delta}$ as a non-redundant disjunction of conjunctions of literals $\vee_{i=1}^{\ell} \wedge_{j \in T_{i}} p_{i}(\beta)$;

- create $\ell$ weighted products $\left[\wedge_{j \in T_{i}} p_{i}(\beta), \beta\right]$;

- delete subsumed weighted products.

Then the fuzzy measure $\mu$ is such that its qualitative Möbius transform is defined by $\mu_{\#}\left(T_{i}\right)=\beta$ for all weighted products $\wedge_{j \in T_{i}} p_{i}(\beta)$.

\section{Related work}

There do not exist many works trying to provide a logical reading of decision processes. In decision under uncertainty, let us mention a logical approach [5] where uncertain knowledge and prioritized preference are represented by means of two distinct possibilistic logic $N$-bases, and where the pessimistic or optimistic decision criteria that are maximized are particular cases of Sugeno integrals. In [2], a multicriteria decision problem is expressed in terms of prioritized constraints and wishes, where constraints are modelled by an $N$-base, and wishes by a $\Delta$-base, handled separately in the decision process; however the latter is not interpreted as an $N$-based put in disjunctive form. In [13], in a multiple criteria decision perspective, a qualitative approach, also in the spirit of possibilistic logic, is compared to a counterpart of a Choquet integral-based aggregation process. However, the closest related work is [16] in which Greco et al. show that the set of alternatives for which a Sugeno integral is less than a given score $\gamma$ can be described by means of if-then rules. 
More precisely, Greco et al. consider a slightly different setting from the one in this paper. Let us give a brief refresher of their result.

Let us begin with the notations. For each $i \in \mathcal{P}, L_{i}$ is the set of ratings of alternatives with respect to criteria $i$. The set of alternatives is thus $X=\Pi_{i=1}^{n} L_{i}$. On $X$ there is a comprehensive weak preference relation $\succeq$. For all $x, y \in X$ $x \succeq y$ means $x$ is at least as good as $y$. Its asymmetric part is denoted by $\succ$.

In such a context, there exists the following link between the Sugeno integral representation and a set of decision rules (after Theorem 3 in [16]):

\section{Proposition 10. A preference relation $\succeq$ on $X$ is representable by a Sugeno integral if and only if there exist}

- functions $g_{i}: L_{i} \rightarrow[0,1]$ for all $i=1, \ldots, n$ called criteria;

- a function $g: X \rightarrow[0,1]$ called comprehensive evaluation;

- a set of decision rules, called single-graded, of the form "if $g_{i_{1}}\left(x_{i_{1}}\right) \geq g(y), \ldots, g_{i_{k}}\left(x_{i_{k}}\right) \geq g(y)$, then $x \succeq y$ ", where $\left\{i_{1}, \ldots, i_{k}\right\} \subseteq\{1, \ldots, n\}$ such that

- each $z \in X$ such that $z \geq y$ satisfies the antecedent of at least one rule whose consequent is "then $x \geq w$ " where $w \succeq y$

- each $z \in X$ such that $\neg(z \succeq y)$ does not satisfy the antecedent of any rule whose consequent is "then $x \succeq w$ " with $w \succeq y$;

○ if the decision rule: "if $g_{i_{1}}\left(x_{i_{1}}\right) \geq g(y), \ldots, g_{i_{k}}\left(x_{i_{k}}\right) \geq g(y)$ then $x \geq y$ " holds, then for any $w \in X$ such that $g(y) \geq g(w)$, the decision rule "if $g_{i_{1}}\left(x_{i_{1}}\right) \geq g(w), \ldots, g_{i_{k}}\left(x_{i_{k}}\right) \geq g(w)$ then $x \geq w$ " also holds.

In a nutshell, the result explains that the decision rules representable by a Sugeno integral are only single-graded rules, i.e., that involve a single threshold $g(y)$. But Greco et al. [16] do not provide an explicit representation of a Sugeno integral in $N$-based, or $\Delta$-based possibilistic logic.

Similar to our work, Greco et al. consider sets of the form $\left\{x \mid \oint_{\mu}(x) \geq \gamma\right\}$. More precisely, for an alternative $x$ and a real number $\gamma$, the rules proposed by Greco et al. can thus be expressed as follows, letting $\gamma=g(y)$ for some fixed option $y$. Let $A(x, \gamma)$ be the set of criteria on which $x$ is better or equal to $\gamma$ :

$$
\text { If } \mu(A(x, \gamma)) \geq \gamma \text { then } \oint_{\mu}(x) \geq \gamma
$$

Let us note that using the results presented in [27] the previous condition is necessary and sufficient.

In this paper, we have shown that a Sugeno integral can be modeled by a $\Delta$-base or an $N$-base in possibilistic logic. What the above remarks show is that each single-graded rule in the sense of Greco et al. can thus be viewed as a weighted formula of the form $\left[\wedge_{j \epsilon_{i}} p_{j}(\gamma), \gamma\right]$ in the obtained $\Delta$-base (however the conclusion part of their rules is obtained in our setting by computing the possibility degree of the corresponding alternative, instead of concluding on a pairwise comparison of alternatives). What this paper lays bare is that the $g(y)$ values appearing in each singlegraded rule of Greco et al. can be restricted to qualitative Möbius transform weights associated to the set of criteria appearing in the condition part of the rule. In the future, we shall consider encoding single-graded rules of the form "if $g_{i_{1}}\left(x_{i_{1}}\right) \leq g(y), \ldots, g_{i_{k}}\left(x_{i_{k}}\right) \leq g(y)$, then $x \preceq y$ ", directly in $N$-based possibilistic logic, using the min-max expression of Sugeno integral (that appears in Proposition 1).

\section{Concluding remarks}

Any set of logical formulas not involving negation can be represented by a Boolean polynomial. Sugeno integrals are lattice polynomials obeying boundary conditions. It is then natural to look for a logical description of Sugeno integrals. As the latter involve constants in a totally ordered set, the natural target language is possibilistic logic. This paper has demonstrated this connection in detail and pointed out the relationship with the rule-based representation of Sugeno integrals. The search for a logical account of fuzzy measures is not fully carried out yet, though. The proposed translation enables assertions of the form $\oint_{\mu}(x) \geq \gamma$ to be verified via a kind of model-checking problem (verify whether an interpretation $x$ satisfies a prioritized knowledge base). But it does not allow to represent such assertions at the syntactic level and reason about them. This task may request the use of generalized possibilistic logic [11], and probably its extension where the semantics of formulas is in terms of families of fuzzy measures. 


\section{References}

[1] S. Benferhat, S. Kaci, Logical representation and fusion of prioritized information based on guaranteed possibility measures: application to the distance-based merging of classical bases, Artif. Intell. 148 (2003) 291-333.

[2] S. Benferhat, D. Dubois, S. Kaci, H. Prade, Bipolar possibility theory in preference modeling: representation, fusion and optimal solutions, Inf. Fusion 7 (2006) 135-150.

[3] G. Choquet, Theory of capacities, Ann. Inst. Fourier 5 (1953) 131-295.

[4] M. Couceiro, J.-L. Marichal, Characterizations of discrete Sugeno integrals as polynomial functions over distributive lattices, Fuzzy Sets Syst. 161 (2010) 694-707.

[5] D. Dubois, D. Le Berre, H. Prade, R. Sabbadin, Using possibilistic logic for modeling qualitative decision: ATMS-based algorithms, Fund. Inf. 37 (1-2) (1999) 1-30.

[6] D. Dubois, J.-L. Marichal, H. Prade, M. Roubens, R. Sabbadin, The use of the discrete Sugeno integral in decision-making: a survey, Int. J. Uncertain. Fuzziness Knowl. Based Syst. 9 (2001) 539-561.

[7] D. Dubois, H. Prade, Possibility theory as a basis for preference propagation in automated reasoning, in: Proceedings of the First IEEE International Conference on Fuzzy Systems 1992 (FUZZ-IEEE'92), San Diego, CA, March 8-12, 1992, pp. 821-832.

[8] D. Dubois, H. Prade, Possibility theory: qualitative and quantitative aspects, in: P. Smets (Ed.), Handbook on Defeasible Reasoning and Uncertainty Management Systems, Quantified Representation of Uncertainty and Imprecision, vol. 1, Kluwer Academic Publishers, Dordrecht, The Netherlands, 1998, pp. 169-226.

[9] D. Dubois, H. Prade, Qualitative possibility functions and integrals, in: E. Pap (Ed.), Handbook of Measure Theory, vol. 2, Elsevier, Amsterdam, 2002, pp. 1469-1521.

[10] D. Dubois, H. Prade, Possibilistic logic: a retrospective and prospective view, Fuzzy Sets Syst. 144 (2004) 3-23.

[11] D. Dubois, H. Prade, Generalized possibilistic logic, in: S. Benferhat, J. Grant (Eds.), Proceedings of the Fifth International Conference on Scalable Uncertainty Management (SUM'11), Dayton, Ohio, Lecture Notes in Computer Science, vol. 29, Springer, Berlin, 2011, pp. $428-432$.

[12] D. Dubois, H. Prade, A. Rico, A possibilistic logic view of Sugeno integrals, in: P. Melo-Pinto et al. (Ed.), Proceedings of the Eurofuse Workshop on Fuzzy Methods for Knowledge-Based Systems (EUROFUSE'11), Advances in Intelligent and Soft Computing, Régua, Portugal, vol. 107, Springer, Berlin, 2011, pp. 19-30.

[13] R. Gérard, S. Kaci, H. Prade, Ranking alternatives on the basis of generic constraints and examples—a possibilistic approach, in: M.M. Veloso (Ed.), Proceedings of the 20th International Joint Conference on Artificial Intelligence (IJCAI 2007), Hyderabad, January 6-12, 2007, pp. 393-398.

[14] M. Grabisch, The Möbius transform on symmetric ordered structures and its application to capacities on finite sets, Discret. Math. 287 (2004) $17-34$.

[15] M. Grabisch, C. Labreuche, A decade of application of the Choquet and Sugeno integrals in multi-criteria decision aid, Ann. Oper. Res. 175 (2010) 247-286

[16] S. Greco, B. Matarazzo, R. Slowinski, Axiomatic characterization of a general utility function and its particular cases in terms of conjoint measurement and rough-set decision rules, Eur. J. Oper. Res. 158 (2004) 271-292.

[17] A. Kandel, W.J. Byatt, Fuzzy sets, fuzzy algebra, and fuzzy statistics, Proc. IEEE 66 (1978) 1619-1639.

[18] C. Labreuche, A general framework for explaining the results of a multi-attribute preference model, Artif. Intell. 175 (2011) $1410-1448$.

[19] J.-L. Marichal, Aggregation Operations for Multicriteria Decision Aid (Ph.D. thesis), University of Liège, Belgium, 1998.

[20] J.-L. Marichal, On Sugeno integral as an aggregation function, Fuzzy Sets Syst. 114 (3) (2000) 347-365.

[21] R. Mesiar, k-order Pan-discrete fuzzy measures, in: Proceedings of the Seventh International Fuzzy Systems Association World Congress (IFSA'97), Prague, vol. 1, June 25-29, 1997, pp. 488-490.

[22] G. Shafer, A Mathematical Theory of Evidence, Princeton University Press, Princeton, N.J., 1976.

[23] M. Sugeno, Theory of fuzzy integrals and its applications (Ph.D. thesis), Tokyo Institute of Technology, Tokyo, 1974.

[24] M. Sugeno, Fuzzy measures and fuzzy integrals: a survey, in: M.M. Gupta, G.N. Saridis, B.R. Gaines (Eds.), Fuzzy Automata and Decision Processes, North-Holland, Amsterdam, 1977, pp. 89-102.

[25] H. Prade, A. Rico, M. Serrurier, Elicitation of Sugeno integrals: a version space learning perspective, in: J. Rauch, Z.W. Ras, P. Berka, T. Elomaa (Eds.), Proceedings of the 18th International Symposium on Methodologies for Intelligent Systems (ISMIS'09), Prague, Lecture Notes in Artificial Intelligence, vol. 5722, Springer, Berlin, September 14-17, 2009, pp. 392-401.

[26] H. Prade, A. Rico, M. Serrurier, E. Raufaste, Elicitating Sugeno integrals: methodology and a case study, in: C. Sossai, G. Chemello (Eds.), Proceedings of the 10th European Conference on Symbolic and Quantitative Approaches to Reasoning with Uncertainty (ECSQARU'09), Verona, Lecture Notes in Artificial Intelligence, vol. 5590, Springer, Berlin, July 1-3, 2009, pp. 712-723.

[27] A. Rico, M. Grabisch, Ch. Labreuche, A. Chateauneuf, Preference modeling on totally ordered sets by the Sugeno integral, Discret. Appl. Math. 147 (2005) 113-124. 